\title{
A Precise Measurement of the Oxygen Isotope Effect on the Néel Temperature in Cuprates
}

\author{
E. Amit, ${ }^{1}$ A. Keren, ${ }^{1}$ J. S. Lord, ${ }^{2}$ and P. King ${ }^{2}$ \\ ${ }^{1}$ Physics Department, Technion-Israel Institute of Technology, Haifa 32000, Israel \\ ${ }^{2}$ Rutherford Appleton Laboratory, Chilton Didcot, Oxfordshire OX11 OQX, UK
}

Correspondence should be addressed to E. Amit, eranamita@gmail.com

Received 29 December 2010; Accepted 8 March 2011

Academic Editor: Ashok Chatterjee

Copyright () 2011 E. Amit et al. This is an open access article distributed under the Creative Commons Attribution License, which permits unrestricted use, distribution, and reproduction in any medium, provided the original work is properly cited.

\begin{abstract}
A limiting factor in the ability to interpret isotope effect measurements in cuprates is the absence of sufficiently accurate data for the whole phase diagram; there is precise data for $T_{c}$, but not for the antiferromagnetic transition temperature $T_{N}$. Extreme sensitivity of $T_{N}$ to small changes in the amount of oxygen in the sample is the major problem. This problem is solved here by using the novel compound $\left(\mathrm{Ca}_{0.1} \mathrm{La}_{0.9}\right)\left(\mathrm{Ba}_{1.65} \mathrm{La}_{0.35}\right) \mathrm{Cu}_{3} \mathrm{O}_{y}$ for which there is a region where $T_{N}$ is independent of oxygen doping. Meticulous measurements of $T_{N}$ for samples with ${ }^{16} \mathrm{O}$ and ${ }^{18} \mathrm{O}$ find the absence of an oxygen isotope effect on $T_{N}$ with unprecedented accuracy. A possible interpretation of our finding and existing data is that isotope substitution affects the normal state charge carrier density.
\end{abstract}

Isotope substitution is a powerful experimental tool used to investigate complex systems. Ideally, the isotope substitution affects only one parameter, for example, a phonon frequency which is directly related to the nuclear mass. However, the strong coupling of many parameters in cuprates highly limit the ability to interpret isotope effect (IE) experiments. Even though the oxygen isotope substitution is known to affect the superconductivity transition temperatures $T_{c}$, it is unclear whether it primarily impacts phonons, polarons, magnons, doping, or other physical properties [1].

The isotope effect is usually described using the isotope coefficient $\alpha$ via the relation

$$
T_{q} \propto M^{-\alpha_{q}}
$$

where $T_{q}$ is a phase transition temperature, $M$ is the isotope mass and $q=C, N$, and $g$ for the superconducting (SC), antiferromagnetic (AFM), and spin glass critical temperatures, respectively. In many conventional superconductors $\alpha$ was found to be very close to $0.5[2,3]$. The explanation of this $\alpha$ in terms of Cooper pairs glued by phonons was one of the triumphs of the BCS theory for metallic superconductors [4].

In cuprates the isotope effect is much more complicated and $\alpha$ is not single valued and varies across the phase diagram. The consensus today for YBCO like compounds is that in the SC phase, close to optimal doping,

$$
\alpha_{C}^{\mathrm{od}}=0.018 \pm 0.005,
$$

for oxygen substitution [5]. On the SC dome $\alpha_{C}$ increases as the doping decreases $[6,7]$. In the glassy state less data is available, but it seems that the isotope effect reverses sign and $\alpha_{g}$ becomes negative. In extremely underdoped samples, where long range AFM order prevails at low temperatures, data is scarce, controversial, and has relatively large error bars $[5,8]$. The most recent measurements with $\mathrm{Y}_{y} \mathrm{Pr}_{1-y} \mathrm{Ba}_{2} \mathrm{Cu}_{3} \mathrm{O}_{7-\delta}$ show that $\alpha_{N}=0.02$ (3) in the parent compound [5]. There are also several theories dealing with the variation of $\alpha$ along the phase diagram [9-14], but since $\alpha_{C}^{\text {od }}$ and $\alpha_{N}$ are within an error bar of each other one cannot contrast these theories with experiments. In particular, it is impossible to tell whether the same glue that holds the spins together holds the cooper pair together, or not. Increasing the accuracy of the IE measurements of the Néel temperature will shed light on the role of isotope substitution.

As mentioned above, a major limitation on the measurements of $\alpha_{N}$ is the strong dependence of $T_{N}$ on doping. For example, in $\mathrm{Y}_{y} \mathrm{Pr}_{1-y} \mathrm{Ba}_{2} \mathrm{Cu}_{3} \mathrm{O}_{7-\delta}, T_{N}$ decreases with 


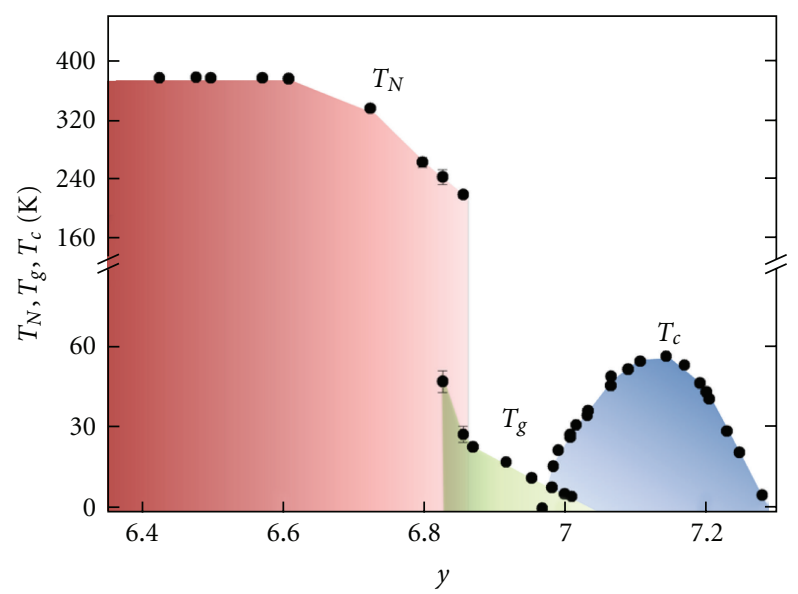

Figure 1: The $\left(\mathrm{Ca}_{0.1} \mathrm{La}_{0.9}\right)\left(\mathrm{Ba}_{1.65} \mathrm{La}_{0.35}\right) \mathrm{Cu}_{3} \mathrm{O}_{y}$ phase diagram [15]. The antiferromagnetic, spin glass, and superconducting phases are represented in red, green, and blue, respectively. For $y<6.6$ the Néel temperature does not depend on $y$. This enables accurate measurements of the oxygen isotope effect on the Néel temperature $T_{N}$.

increasing doping at a rate $\Delta T_{N}=2.5 \mathrm{~K}$ per $\Delta y=0.01$. This strong temperature dependence is, of course, common to many other cuprates. As a consequence, it is very difficult to prepare two samples with exactly the same $T_{N}$ even with the same isotope. The smallest fluctuation in either $y$ or $\delta$ may lead to a huge fluctuation in $T_{N}$ regardless of the isotope effect. This is not the case for $\left(\mathrm{Ca}_{0.1} \mathrm{La}_{0.9}\right)\left(\mathrm{Ba}_{1.65} \mathrm{La}_{0.35}\right) \mathrm{Cu}_{3} \mathrm{O}_{y}$ where $T_{N}$ is constant for oxygen density $y<6.6$, as can be seen in Figure 1 [15].

For our experiments, four sintered pellets were prepared using standard techniques [16]. Two of the pellets were enriched with ${ }^{18} \mathrm{O}$ isotope and two with ${ }^{16} \mathrm{O}$ isotope in the same procedure: the samples were placed simultaneously in two closed tubes, each with different isotope gas, and then they were heated to allow the isotope to diffuse into the sample. In order to achieve a higher percentage of gas, the enrichment was repeated several times.

The ${ }^{18} \mathrm{O}$ isotope content in the samples was determined based on measurements of gas composition being in equilibrium with the sample during the exchange. Balzers Prisma mass spectrometer was used to analyze in situ isotopic composition of the atmosphere. After the exchange process was performed, the weight increase of the sample was also determined as the light ${ }^{16} \mathrm{O}$ isotope was exchanged with the heavy ${ }^{18} \mathrm{O}$. The isotope enrichment in the samples measured by both methods looked to be higher than $80 \%$. Finally a Thermal Analysis (TA) experiment was performed for the investigated samples after all experiments described in this work were fulfilled in order to verify the isotope enrichment. The samples were heated up to $1200^{\circ} \mathrm{C}$ in the NETSCH STA 449C Jupiter analyzer in a stream of helium. During the TA experiments, ion current signals for the ${ }^{18} \mathrm{O}_{2}$, ${ }^{18} \mathrm{O}^{16} \mathrm{O}$, and ${ }^{16} \mathrm{O}_{2}$ molecules were measured using a mass spectrometer (ThermoStar Pfeiffer Vacuum). The results are shown in Figure 2. The isotope content deduced from

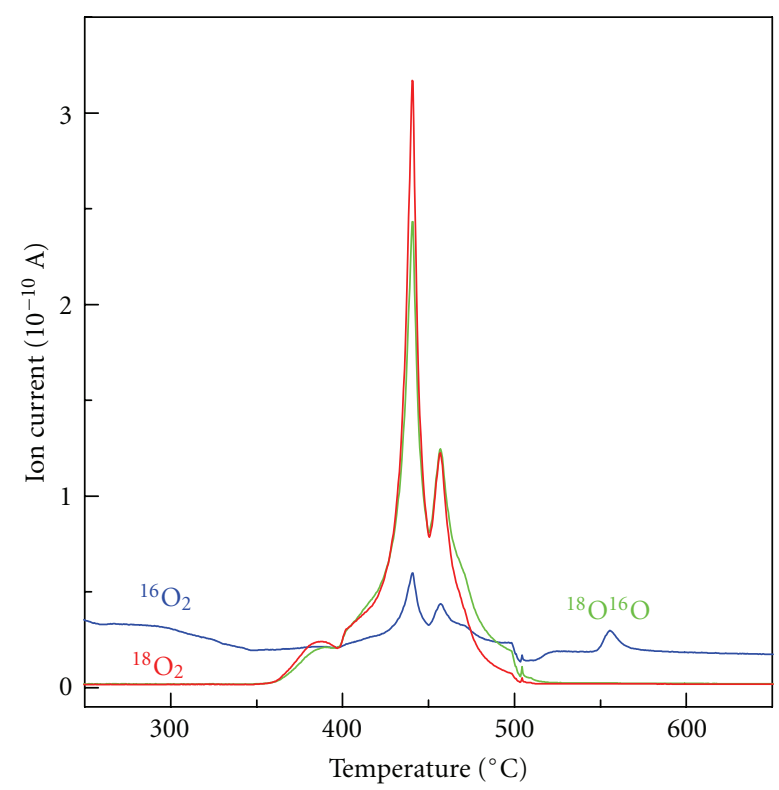

Figure 2: Ion current signals for the ${ }^{18} \mathrm{O}_{2},{ }^{18} \mathrm{O}^{16} \mathrm{O}$, and ${ }^{16} \mathrm{O}_{2}$ molecules (red, green, and blue color, resp.) obtained during heating of the sample in a stream of helium. This graph shows that the ${ }^{18} \mathrm{O}$ isotope fraction in the samples is bigger than $70 \%$ (see text).

these measurements (comparing peak areas for the signals of particular oxygen molecules) was larger than $70 \%$.

The oxygen IE was measured using zero-field muon spin rotation/relaxation ( $\mu \mathrm{SR})$. We particularly used the ISIS facility, which allows low muon relaxation rate and rotation frequency measurements. This is ideal for measurements near magnetic phase transitions where the muon signal varies on a long-time scale. $\mu \mathrm{SR}$ data at temperatures close to the phase transition are shown in Figure 3. As the temperature is lowered from $383.4 \mathrm{~K}$, the relaxation rate increases. At $T=378.5 \mathrm{~K}$ oscillations appear in the data indicating the presence of long range magnetic order. The frequency of oscillations and the relaxation rate increase as the temperature is further lowered. The formula

$$
P_{z}(t)=P_{m}\left(a e^{-\lambda_{1} t}+(1-a) e^{-\lambda_{2} t} \cos (\omega t)\right)+P_{n} e^{-\Delta t}
$$

was fitted to the muon polarization, where $P_{m}, \lambda_{1}, \lambda_{2}$ and $\omega$ are the polarization, relaxation rates, and frequency of muons spin in the fraction of the samples which is magnetic, and $a$ is the weighting factor between the muons experiencing transverse field and longitudinal field. This factor is close to $2 / 3$ and temperature independent. $P_{n}$ and $\Delta$ are the polarization and relaxation of the spin of muons that stopped in the nonmagnetic volume of the sample. The solid lines in Figure 3(a) represent the fits.

The AFM order parameter is determined by the frequency $\omega=\gamma B$, where $B$ is the average magnetic field at muon site and $\gamma$ is the muon gyromagnetic ratio. This frequency can be easily extracted from the $\mu \mathrm{SR}$ data well below the transition but is very difficult to determined near the transition. A second approach is to treat the magnetic volume fraction $P_{m}$ as the order parameter $[5,17] . P_{m}$ can be 


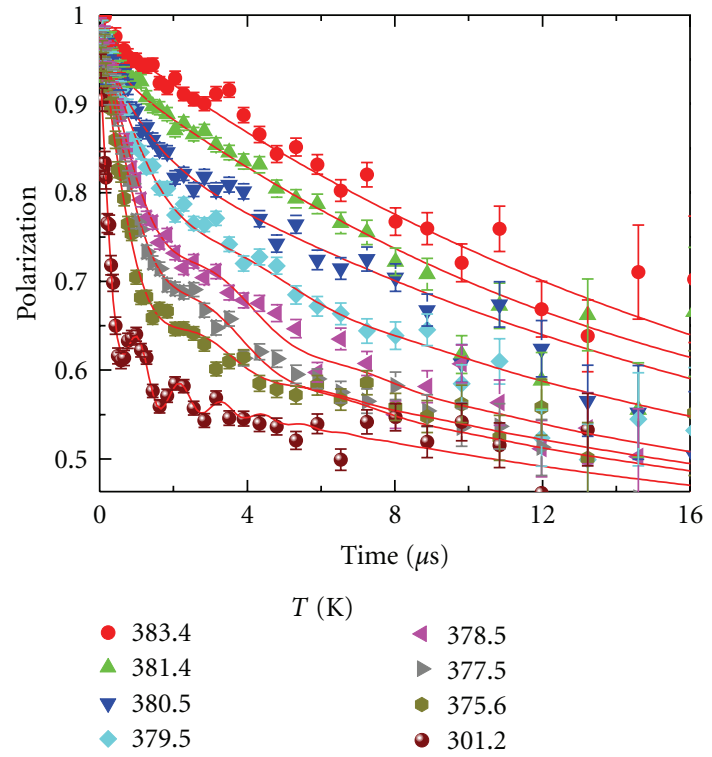

(a)

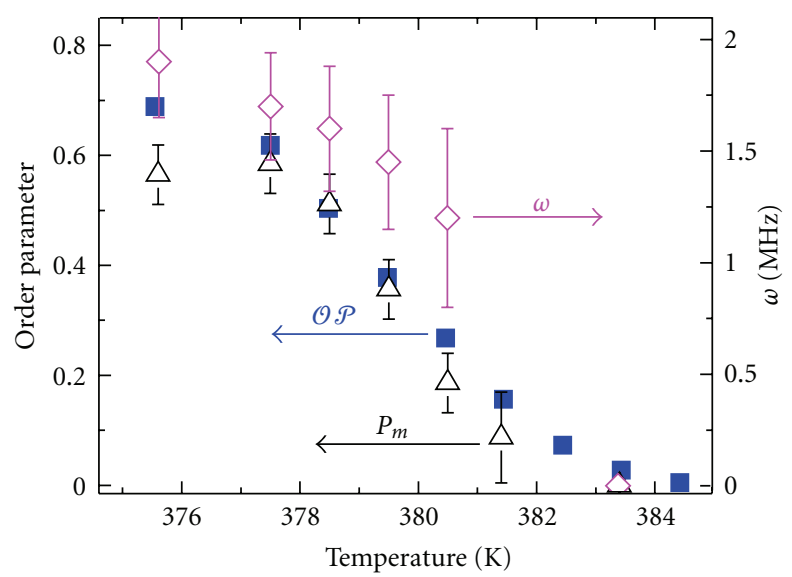

(b)

Figure 3: (a) The $\mu \mathrm{SR}$ raw data at different temperatures and fits of (3) to the data in solid lines. (b) Comparison between three different methods used to describe the AFM phase transition: (i) the muon precession frequency $\omega$ obtained from (3). (ii) the magnetic volume fraction $P_{m}$ determined by (3), and (iii) $\mathcal{O} \mathcal{P}$ calculated by (4).

followed more closely to $T_{N}$, although this parameter also has large error bars when $\omega$ is not well defined. We used an alternative approach similar to [18]; we define an order parameter, which does not require a fit, via the relation

$$
\mathcal{O P}(T) \equiv \frac{\left\langle P_{\text {inf }}\right\rangle-\langle P(T)\rangle}{\left\langle P_{\text {inf }}\right\rangle-\langle P(0)\rangle},
$$

where $\langle P(T)\rangle$ is the average polarization at temperature $T$, and $\left\langle P_{\text {inf }}\right\rangle$ is the average polarization above the transition. The denominator normalizes $\mathcal{O P}$ to 1 at zero temperature. All three order parameters for one sample are shown in Figure 3(b). The transition temperatures determined using the different order parameters are in good agreement. The advantages of $\mathcal{O P}$ are clear: it is a model-free and has very small uncertainties.

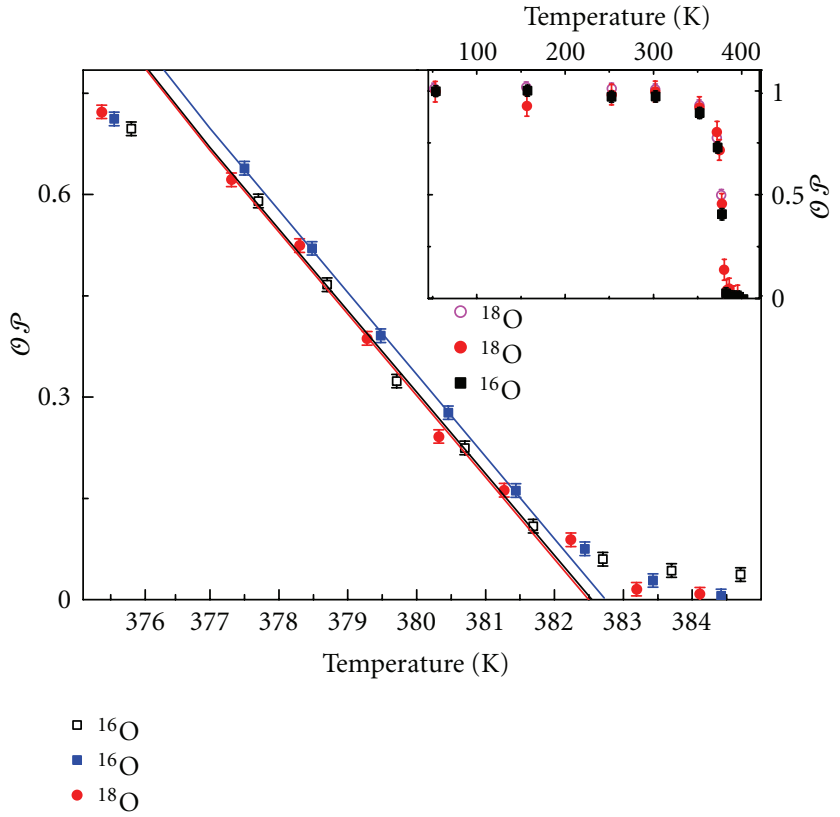

Figure 4: The parameter $\mathcal{O} \mathcal{P}$ (see (4)) versus temperature near $T_{N}$ for ${ }^{18} \mathrm{O}$ and ${ }^{16} \mathrm{O}$ rich samples. The solid lines are fits to the data near the phase transition used to determine $T_{N}$. The inset shows a second experiment on the entire temperature range. No oxygen isotope effect of $T_{N}$ is observed within experimental error.

In Figure 4, we present the $\mathcal{O} \mathcal{P}$ for two samples with ${ }^{16} \mathrm{O}$ and one with ${ }^{18} \mathrm{O}$ around $380 \mathrm{~K}$. A wide temperature range from 50 to $410 \mathrm{~K}$ is shown in the inset for an experiment done on separate occasion, in which two samples of ${ }^{18} \mathrm{O}$ and one with ${ }^{16} \mathrm{O}$ were examined. We determine $T_{N}$ by fitting a straight line to the data in the main panel of Figure 4 in the temperature range 378 to $382 \mathrm{~K}$, for each sample, and taking the crossing with the temperature axis. We find that $T_{N}^{18}=382.49(0.34) \mathrm{K}$ and $T_{N}^{16}=382.64(0.29) \mathrm{K}$. For $100 \%$ isotope substitution the isotope exponent is determined by

$$
\alpha_{N}=-\frac{M_{o}^{16}}{T_{N}^{16}} \frac{T_{N}^{18}-T_{N}^{16}}{M_{o}^{18}-M_{o}^{16}} .
$$

When taking into account the isotopic fraction in the samples we obtain

$$
\alpha_{N}=0.005 \pm 0.011
$$

This result indicates that $\alpha_{N}<\alpha_{C}^{\text {od }}$ (see (2)) beyond the error bars and is consistent with no isotope effect on $T_{N}$.

One possible interpretation of these results is that magnetic excitations are not relevant for superconductivity since the isotopes affect $T_{c}$ without affecting $T_{N}$. This approach was presented, for example, by Zhao et al. [9]. They found that samples enriched with ${ }^{18} \mathrm{O}$ have longer penetration depth $\lambda$ than samples enriched with ${ }^{16} \mathrm{O}$ with the same amount of oxygen per unit cell. $\lambda$ is related to the SC carrier density $n_{s}$ and effective mass $m^{*}$ by $\lambda^{-2} \propto n_{s} / m^{*}$ so a priory both $n_{s}$ and $m^{*}$ can be affected by isotope substitution [19]. They ruled out the possibility that the number of carriers 


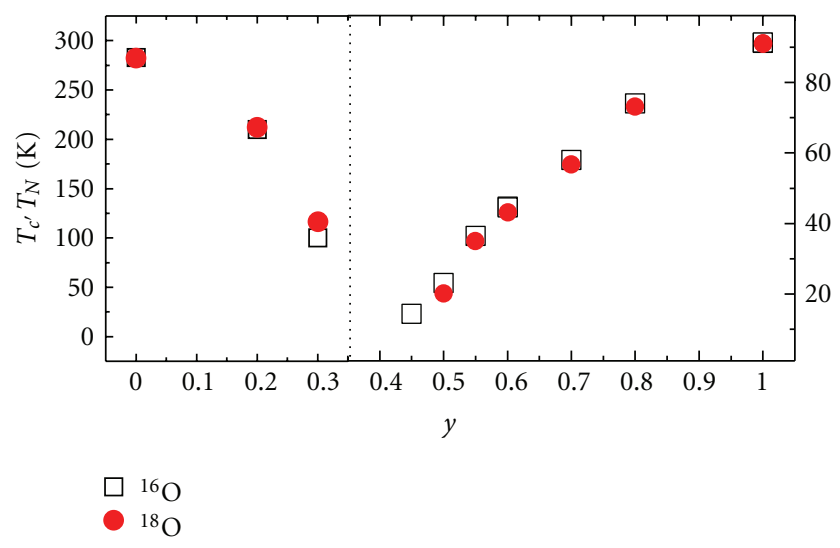

(a)

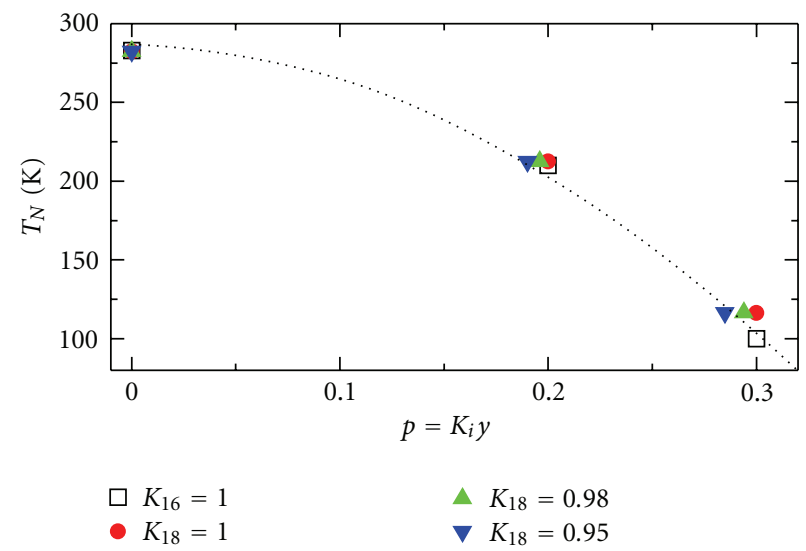

(b)

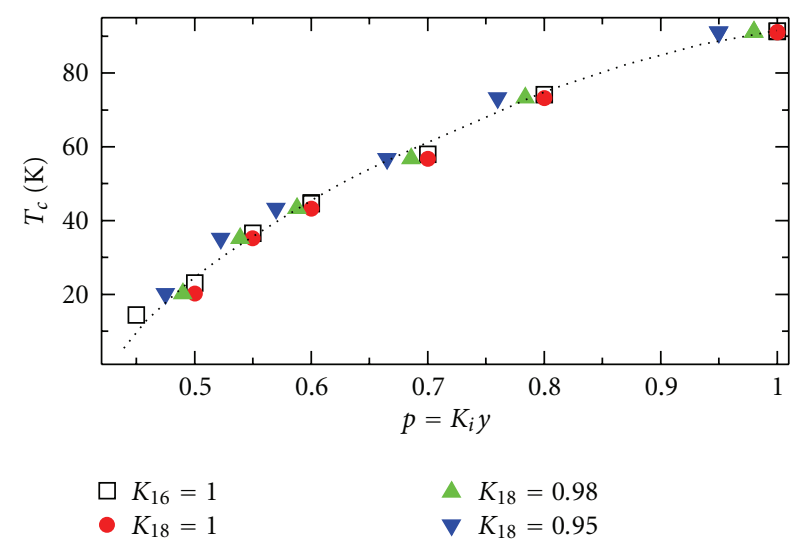

(c)

Figure 5: (a) Oxygen isotope effect of $\mathrm{T}_{N}$ (left) and $\mathrm{T}_{c}$ (right) in $\mathrm{Y}_{y} \mathrm{Pr}_{1-y} \mathrm{Ba}_{2} \mathrm{Cu}_{3} \mathrm{O}_{7-\delta}$ taken from [5]. The ${ }^{18} \mathrm{O}$ and ${ }^{16} \mathrm{O}$ samples are in filled red circles and empty black squares, respectively. Figures (b) and (c) are demonstration of the IE using charge carriers density $p=K_{i} y$, instead of oxygen content $y$. The values of $K_{i}$ are given in the figures. Green triangles $\left(K_{i}=0.98\right)$ represent reduction of $2 \%$ in the number of charge carriers in the ${ }^{18} \mathrm{O}$ samples compared to the ${ }^{16} \mathrm{O}$ sample. In this case both $T_{N}$ and $T_{c}$ are functions of $p$ regardless of the isotope. concentration varies by demonstrating that the thermal expansion coefficient of samples with different isotopes are the same. The authors therefore concluded that the IE changes the mass of the cooper pairs, which could be explained by polaronic supercarriers.

An alternative interpretation is that the isotopes affect the efficiency of doping, as suggested in [13]. To demonstrate this interpretation we present in Figure 5(a) the critical temperatures in $\mathrm{Y}_{y} \mathrm{Pr}_{1-y} \mathrm{Ba}_{2} \mathrm{Cu}_{3} \mathrm{O}_{7-\delta}$ for the two different isotopes taken from [5]. $T_{N}^{18}$ seems to be a bit higher than $T_{N}^{16}$, but $T_{c}^{18}$ is a bit lower than $T_{c}^{16}$. However, if we define an efficiency parameter $K_{i}$ which relates the number of holes $p$ to the number of oxygen ions in the unit cell $y$ via $p=$ $K_{i} y$, where $i$ stands for the isotope type, we can generate a unified phase diagram. This is demonstrated in Figures 5(b) and 5(c) for $T_{N}$ and $T_{c}$, respectively. In these graphs three different values of $K_{18}$ are used to generate $p$ while keeping $K_{16}=1$. When using $K_{18}=0.98$, both curves of $T_{N}$ and $T_{c}$ versus $p$ for the two different isotopes collapse to the same curve. Similar scaling of the doping axis was applied to the $\left(\mathrm{Ca}_{x} \mathrm{La}_{1-x}\right)\left(\mathrm{Ba}_{1.75-x} \mathrm{La}_{0.25+x}\right) \mathrm{Cu}_{3} \mathrm{O}_{y}$ system [15] and was explained by NQR [20].

Next we discuss the IE on the stiffness in the above scenario. We assume that the effective mass of the SC charge carrier $m^{*}$, the critical $p$ where superconductivity starts $p_{\text {crit }}$, and where $T_{c}$ is optimal $p_{\text {opt }}$, are not affected by the isotope substitution. The stiffness can be measured by the muon transverse relaxation rate $\sigma$, and it is expected that [19]

$$
\sigma^{i}=C\left(p^{i}-p_{\text {crit }}\right),
$$

where $C$ is a constant. Dividing the differential of $\sigma$ from (7) by the relaxation at optimal doping yields

$$
\frac{d \sigma}{\sigma_{\mathrm{opt}}}=\frac{d p}{p_{\mathrm{opt}}-p_{\text {crit }}}=\frac{y\left(K_{16}-K_{18}\right)}{p_{\mathrm{opt}}-p_{\text {crit }}} .
$$

The expected change in the stiffness due to isotope substitution can be calculated from (8) using $p_{\mathrm{opt}}=1$, $p_{\text {crit }}=0.42$ (which are extracted from Figure 5(c)), and $\sigma_{\text {opt }}=3.0 \mu \mathrm{s}^{-1}$ [21]. For $y=0.8, K_{16}=1$ and $K_{18}=0.98$ we get $d \sigma=0.083 \mu^{-1}$. This value is consistent with the experimental value of $d \sigma=0.08 \mu \mathrm{s}^{-1}$ reported in [21]. In other words a $2 \%$ difference in the doping efficiency between the two isotopes can explain both the variations in the phase diagram and the variation in the stiffness.

Our experiment shows that oxygen isotope substitution does not affect the Néel temperature and therefore does not play a role in magnetic excitations. However, the isotope effect of $T_{c}$ does not necessarily imply that phonons play a role in cuprate superconductivity. We show that an isotopedependent doping efficiency can explain the variation in $T_{c}$ and in the magnetic penetration depth between samples rich in ${ }^{16} \mathrm{O}$ or ${ }^{18} \mathrm{O}$.

\section{Acknowledgments}

The authors would like to thank the ISIS pulsed muon facility at Rutherford Appleton Laboratory, UK for excellent 
muon beam conditions. This work was funded by the Israeli Science Foundation, the joint German-Israeli DIP project, and the Posnansky research fund in high temperature superconductivity.

\section{References}

[1] P. A. Lee, "From high temperature superconductivity to quantum spin liquid: progress in strong correlation physics," Reports on Progress in Physics, vol. 71, no. 1, Article ID 012501, 2008.

[2] E. Maxwell, "Isotope effect in the superconductivity of mercury," Physical Review, vol. 78, no. 4, p. 477, 1950.

[3] C. A. Reynolds, B. Serin, W. H. Wright, and L. B. Nesbitt, "Superconductivity of isotopes of mercury," Physical Review, vol. 78, no. 4, p. 487, 1950.

[4] J. Bardeen, L. N. Cooper, and J. R. Schrieffer, "Theory of superconductivity," Physical Review, vol. 108, no. 5, pp. 11751204, 1957.

[5] R. Khasanov, A. Shengelaya, D. Di Castro et al., "Oxygen isotope effects on the superconducting transition and magnetic states within the phase diagram of $\mathrm{Y}_{1-\mathrm{x}} \mathrm{Pr}_{\mathrm{x}} \mathrm{Ba}_{2} \mathrm{Cu}_{3} \mathrm{O}_{7-\delta}$," Physical Review Letters, vol. 101, no. 7, Article ID 077001, 2008.

[6] D. J. Pringle, G. V. M. Williams, and J. L. Tallon, "Effect of doping and impurities on the oxygen isotope effect in hightemperature superconducting cuprates," Physical Review B, vol. 62, no. 18, pp. 12527-12533, 2000.

[7] H. Keller, Unconventional Isotope Effects in Cuprate Superconductors, Springer, Berlin, Germany, 2005.

[8] G. M. Zhao, K. K. Singh, and D. E. Morris, "Oxygen isotope effect on Néel temperature in various antiferromagnetic cuprates," Physical Review B, vol. 50, no. 6, pp. 4112-4117, 1994.

[9] G. M. Zhao, M. B. Hunt, H. Keller, and K. A. Müller, "Evidence for polaronic supercarriers in the copper oxide superconductors $\mathrm{La}_{2-X} \mathrm{Sr}_{X} \mathrm{CuO}_{4}$," Nature, vol. 385, no. 6613, pp. 236-238, 1997.

[10] A. Bill, V. Z. Kresin, and S. A. Wolf, "The isotope effect in superconductors," http://arxiv.org/abs/cond-mat/9801222.

[11] J. F. Baugher, P. C. Taylor, T. Oja, and P. J. Bray, "Nuclear magnetic resonance powder patterns in the presence of completely asymmetric quadrupole and chemical shift effects: application to metavanadates," Journal of Chemical Physics, vol. 50, no. 11, article 4914, 12 pages, 1969.

[12] D. S. Fisher, A. J. Millis, B. Shraiman, and R. N. Bhatt, "Zeropoint motion and the isotope effect in oxide superconductors," Physical Review Letters, vol. 61, no. 4, p. 482, 1988.

[13] V. Z. Kresin and S. A. Wolf, "Microscopic model for the isotope effect in the high- $T_{c}$ oxides," Physical Review B, vol. 49, no. 5, pp. 3652-3654, 1994.

[14] M. Serbyn and P. A. Lee, "Isotope effect on the superfluid density in conventional and high-temperature superconductors," Physical Review B, vol. 83, no. 2, Article ID 024506, 8 pages, 2011.

[15] R. Ofer, G. Bazalitsky, A. Kanigel et al., "Magnetic analog of the isotope effect in cuprates," Physical Review B, vol. 74, no. 22, Article ID 220508, 4 pages, 2006.

[16] D. Goldschmidt, G. M. Reisner, Y. Direktovitch et al., "Tetragonal superconducting family $\left(\mathrm{Ca}_{x} \mathrm{La}_{1-x}\right)$ $\left(\mathrm{Ba}_{1.75-x} \mathrm{La}_{0.25+x}\right) \mathrm{Cu}_{3} \mathrm{O}_{y}$ : the effect of cosubstitution on the transition temperature," Physical Review B, vol. 48, no. 1, pp. 532-542, 1993.

[17] R. Ofer, A. Keren, O. Chmaissem, and A. Amato, "Universal doping dependence of the ground-state staggered magnetization of cuprate superconductors," Physical Review B, vol. 78, no. 14, Article ID 140508, 2008.

[18] M. Shay, A. Keren, G. Koren et al., "Interaction between the magnetic and superconducting order parameters in a La1.94 $\mathrm{Sr} 0.06 \mathrm{CuO} 4$ wire studied via muon spin rotation," Physical Review B, vol. 80, no. 14, Article ID 144511, 2009.

[19] Y. J. Uemura, G. M. Luke, B. J. Sternlieb et al., "Universal correlations between $T_{c}$ and $n_{s} / m^{*}$ (Carrier density over effective mass) in High- $T_{c}$ cuprate cuperconductors," Physical Review Letters, vol. 62, no. 19, pp. 2317-2320, 1989.

[20] E. Amit and A. Keren, "Critical-doping universality for cuprate superconductors: Oxygen nuclear-magnetic-resonance investigation of $\left(\mathrm{Ca}_{x} \mathrm{La}_{1-x}\right)\left(\mathrm{Ba}_{1.75-x} \mathrm{La}_{0.25+x}\right) \mathrm{Cu}_{3} \mathrm{O}_{y}$," Physical Review B, vol. 82, no. 17, Article ID 172509, 4 pages, 2010.

[21] R. Khasanov, A. Shengelaya, K. Conder et al., "Correlation between oxygen isotope effects on transition temperature and magnetic penetration depth in high-temperature superconductors close to optimal doping," Physical Review B, vol. 74, no. 6, Article ID 064504, 6 pages, 2006. 

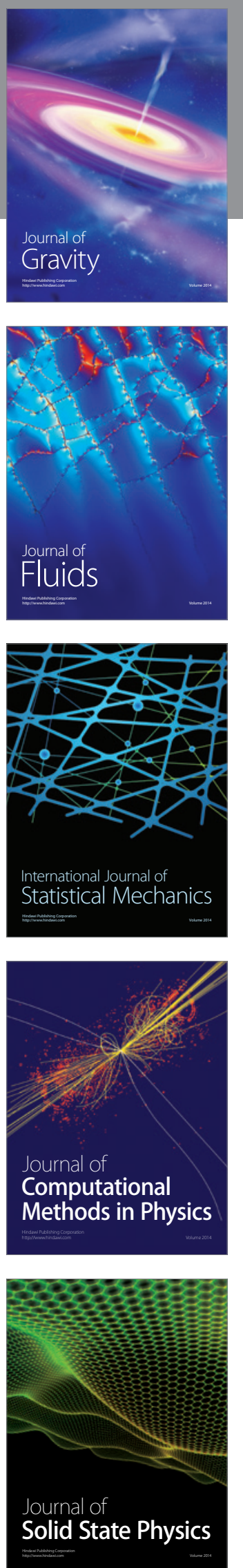

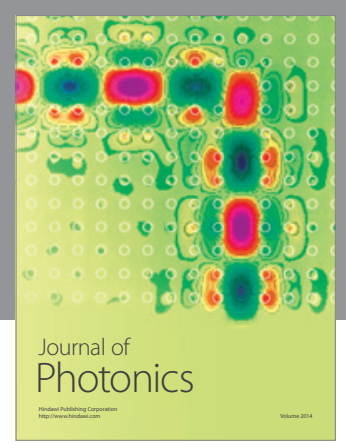

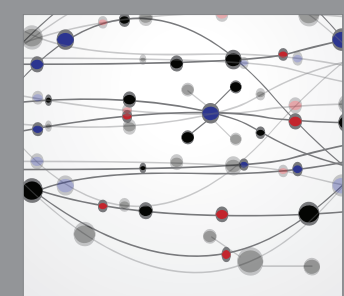

The Scientific World Journal
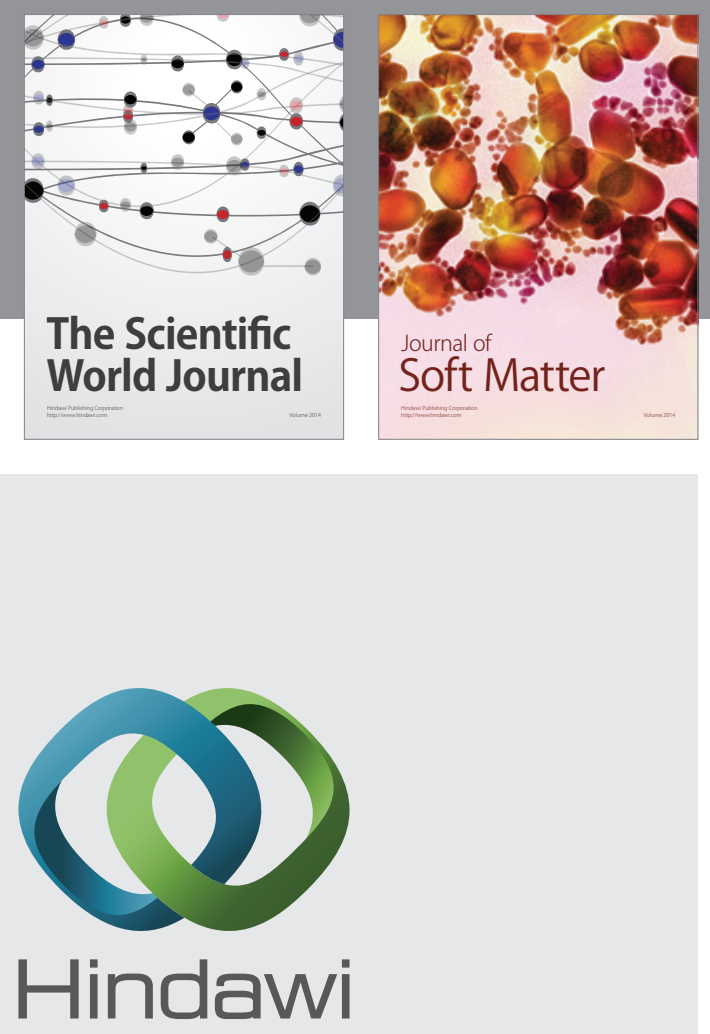

Submit your manuscripts at

http://www.hindawi.com
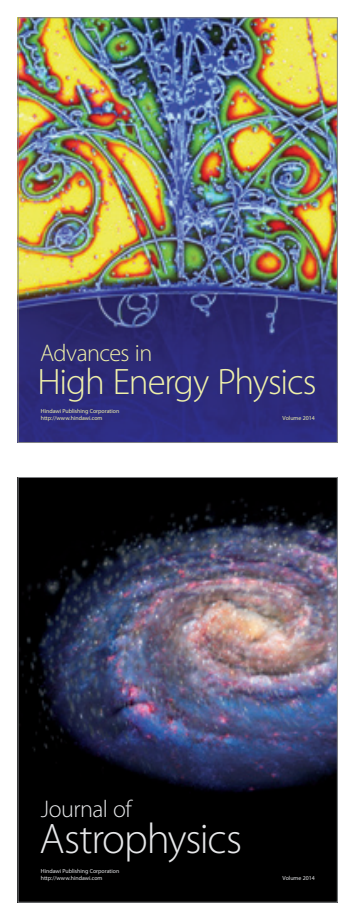
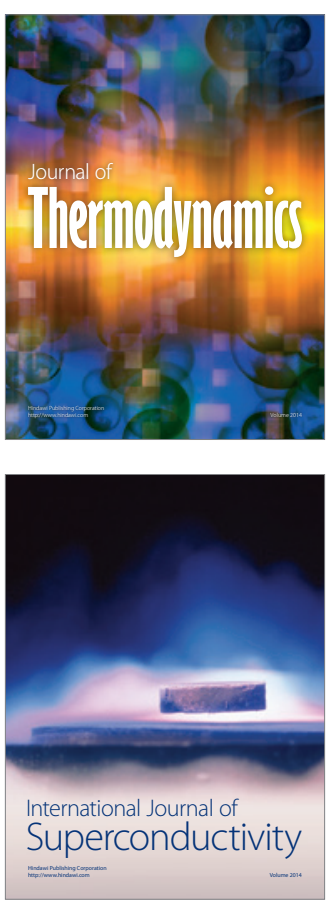
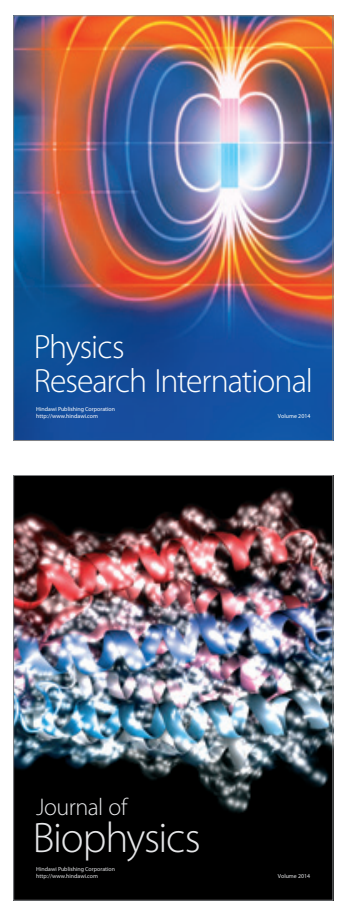
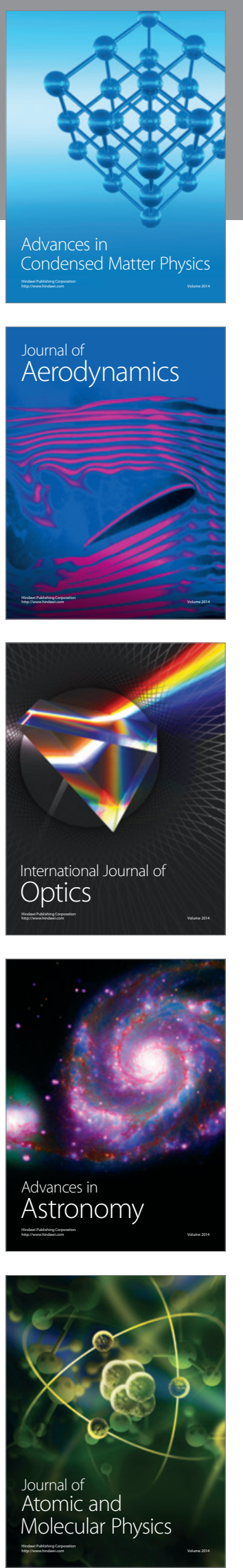